meeting of the International Medical Congress in London, in 1881, it was thought that the meeting of the British Medical Association would probably be unsuccessful if held in that year; but a general opinion was expressed that, in any following year, the Association would be made very welcome.

International Medical Congress, 1881.-The Executive Committee made their report to the General Committee of this Congress, which met at the College of Physicians on 13th July. The officers of the Congress were proposed and nominated. The sections were agreed upon, and the treasurer, Mr. Bowman, announced that large subscriptions had already been received. It was agreed that the time of meeting of the Congress should be from the 3rd to the 9th of August, 1881. The President of the Council of the British Medical Association stated that the Council of that body had postponed their meeting to the following week. It was also announced that the Congress would meet in rooms granted for the purpose by the University of London, the Royal Society, and the other learned societies meeting in Burlington House, so that the sections will be all practically under the same roof.

Invitations to attend the Congress, and to subscribe to its funds, will be shortly issued to all legally qualified medical practitioners in the United Kingdom. Invitations to attend the meeting will be sent to the different countries of Europe, to America, the Colonies, and India. Papers may be read or discussions held in English, in French, or in German; and the volume of Transactions subsequently to be published, will contain the various communications in the language in which they were delivered or read. It is not yet settled, but there is reason to expect that the very highest patronage may be extended to this national undertaking which, it is to be hoped, will form an epoch in the medical history of our country.

\title{
REVIEWS.
}

A Manual and Atlas of Medical Ophthalmoscopy. By W. R. Gowers, M.D. London: J. \& A. Churchill. 1879.

WITH the ophthalmoscope the extremity of the optic nerve and its expansion, the retina, with its blood-vessels, can be seen, and the pathological changes occurring in those 
structures observed during life, which had hitherto only been revealed on post-mortem examination. Some of those changes originate in, and are limited to the eye; others originating in the eye and extending backwards through the optic nerve may affect more or less the integrity of the parts within the cranium; and lastly, a third class, the result and evidence of morbid processes elsewhere extending to the eye either by direct propagation of the disease through the nervous or vascular system, or by irritation excited by disease in a distant part, communicated to the extremity of the optic nerve by processes not well understood at present. The exceptional liability of the optic nerve to be affected by diseases in other parts of the nervous system, and the distinctive character of the changes to be observed, these frequently afford important information as to the existence and nature of the morbid processes which have excited them.

Assuming that it is possible in all cases to distinguish those diseases of the eye which are the result of morbid processes elsewhere from those which are limited to the eye, the value of the ophthalmoscope in the hands of a competent observer will depend on the relative frequency and persistency of certain well defined and characteristic ophthalmoscopic appearances in connection with a given class of diseases of the nervous or vascular systems. For, if it can be demonstrated that in fourfifths of the cases of tumour of the brain there is engorgement of the optic nerve at some stage of the disease, then, from a clinical point of view, this fact must have important bearings on the diagnosis and prognosis in obscure diseases of the brain. If, on the other hand, engorgement of the optic nerve is found to be present in other diseases of the brain, the real significance and value of the symptom can only be duly appreciated when the common proximate cause has been satisfactorily demonstrated. The diversity of opinion which at present exists on this and kindred points renders further investigations necessary in order that the scope and limits of medical ophthalmoscopy may be accurately defined, and the discredit which the exaggerated expectations which had been raised, and the negative results often obtained have thrown on the whole subject, may be removed, and the real and acknowledged value of the information which it gives properly recognised. In this respect the medical ophthalmoscopy of Dr. Gowers must be regarded as an important contribution to the literature of the subject. For, whilst the work is primarily and essentially a complete treatise on the ophthalmoscope addressed to medical practitioners, the number of carefully reported cases, verified 
by post-mortem examinations, adduced in support of his views, give a strong character of individuality to the work.

The work is divided into two parts. The first, devoted to the consideration of the general pathology of the fundus, executed with great care, gives in the space of 112 pages a complete and exhaustive account of what is known up to the present time of this obscure and difficult subject. On account of the low magnifying power ( 4 to 20 diameters), and the peculiarity of the illumination under which objects in the fundus of the eye are observed, it is impossible in most cases to obtain more than a general view of parts, many of which require the highest powers of the microscope to resolve, consequently, many of the pathological changes which occur in those tissues escape observation, unless they can be connected in some way with corresponding changes in the general aspect of the parts affected, e.g., opacity, hypertrophy, atrophy, change of tint, \&c. The exceptional opportunities which Dr. Gowers has had of supplementing the ophthalmoscopic appearances by post-mortem examination have done much to clear up some of the more obscure points. But that much remains to be done will be understood and admitted, when it is stated that a fundamental point, such as the existence of inflammation of the optic papilla (papillitis), has been questioned. The optic papilla is the most conspicuous object in the fundus, and being in direct anatomical connection with important parts at the base of the brain, it is the part most liable to be affected by intracranial disease. But if the fact has been fully admitted, the nature of the alterations there observed, and the conditions which are produced, their frequency and clinical value, are still subjects of dispute. By far the most striking of these changes is swelling or engorgement in its different forms. Dr. Gowers recognises three principal forms or varieties, founded purely on their clinical characters.

"1. Slight papillitis-including the condition described as congestion with œedema, in which the changes are so slight as to dim, but not obscure, the edges of the disc on indirect examination, although it may be invisible wholly or in part to direct examination.

" 2. Moderate papillitis. Obscuration of the edge of the disc, or of the affected portion; complete, even to indirect examination; swelling moderate, commonly reddish; veins natural or large, sometimes white tissue about the vessels, close to them or extending on some distance in the disc.

" 3 . Intense papillitis. Great swelling; veins at first large, and arteries small; many hæmorrhages; retina often involved 
by direct damage or by hæmorrhages. Always succeeds a slighter stage in which the evidence of strangulation may be at first little marked."

The relations of optic neuritis to encephalic disease, the mechanism by which the one acts on the other, and the indications which, from a clinical point of view, may be drawn from the presence of one or other of the forms referred to, have attracted the attention of physicians since 1860, when von Graefe first pointed out the connection between tumour of the brain and optic neuritis. Dr. Gowers, after enumerating the different theories which have been advanced-intra-cranial pressure acting on the circulation; pressure of fluid in the sub-dural space, acting along the optic nerve sheaths; accumulation of fluid in the lymph spaces at the optic nerve entrance; irritation of intra-cranial tumour acting on the vaso-motor system; the direct propagation of inflammation along the optic nerve-goes on to say that-

"This remarkable difference of opinion, regarding a fact so fundamental, is a striking proof that much more careful observation is needed of the conditions of origin of optic neuritis, the pathological changes which can be traced in it, and the allied conditions in which it does not arise, before an adequate theory can be framed, or any effective distinction into varieties can be made. The following considerations are intended rather to further this end than to establish any definite theory of its causation.

"The first point to be borne in mind, is that optic neuritis limited to, or at least most intense in, the optic papillæ, may occur without any obvious cranial or cerebral disease.

"The facts of medical ophthalmoscopy certainly make it difficult to connect papillitis with increase of intra-cranial pressure. On the other hand, as I have many times seen in cases of tumour with neuritis, there may be no sign of increased intra-cranial pressure during life, or after death."

Distension of the optic sheath "is certainly very frequent in cases of optic neuritis. It is not, however, as has been alleged, invariable either in cases of cerebral tumour with optic neuritis, or in conditions of increased intra-cranial pressure. It may be absent in tumour cerebri with characteristic neuritis. . . . It may be absent in tumour with internal effusion. Great distension of the lateral ventricles was caused in case 3, by a tumour near the corpora quadrigemina; there was optic neuritis, but with no distension of the sheaths. (In both these cases, however, the 
neuritis was found to be 'descending.') In case 21 of old neuritis, due to a tumour occupying the whole of the third ventricle and interpeduncular space, and extending in front of the optic commissure, and causing enormous distension of the lateral ventricles, the optic sheaths carefully examined in situ were quite empty. They were loose, and had evidently been much distended. This case suggests that pressure at the base of the brain may even be incompatible with continued distension of the sheath. In tubercular meningitis, again, the condition to which the distension of the sheath appears to be related is not distension of the ventricles, or increased intra-cranial pressure, but increase of the sub-arachnoid fluid, and it bears to this disease certainly no relation to the occurrence of neuritis."

The general conclusion arrived at, after a careful survey of the facts, is, "That, in cases of cerebral tumour, evidence of descending neuritis may be traced much more commonly than current statements suggest; while, in cases of meningitis, the evidence of descending neuritis is almost invariable.

"That the resulting papillitis may be, and remains, slight, or may become intense, and present the appearance of mechanical congestion. The causes of this difference we do not know.

"That such mechanical congestion does not, as a rule, result from compression of the vessels in or just behind the sclerotic ring, but always, when intense, from compression by inflammatory products in the substance of the papilla. It must not be forgotten that an increase in the size of the vessels may be of reflex vaso-motor origin, as in all inflamed parts.

"That, while slow increase of intra-cranial pressure has no effect on the retinal vessels, a sudden increase hinders the escape of blood from the eye for a time, and may intensify a papillitis originating in another way.

"That distention of the sheath of the nerve alone is probably insufficient to cause papillitis, but may perhaps intensify the process otherwise set up, leading to retention or augmentation of fluid in the lymphatic spaces in the eye, fluid which may, in some cases, possess an irritative quality.

"In the cases, certainly rarer than is commonly supposed, in which no trace of descending neuritis can be detected, two possibilities may be borne in mind-(1) that the effect of a sudden increased intra-cranial pressure and of distention of the sheath together may possibly be sufficient to set up a papillitis; and (2) that a condition of irritation may be propagated down 
the nerve, which cannot be recognised by the microscope, but which, reaching the papilla, may excite a papillitis."

The frequent association of neuritis with papillitis in tumour of the brain, and the occurrence of distention of the ventricles in two cases reported here, lend some support to the theory recently advanced by Parinand,* that distention of the lateral ventricles, with odema of the brain and optic nerve, is the invariable cause of papillitis or œdema of the optic papilla, which he holds to be the initial stage in tumour of the brain as well as in acute meningitis. Dr. Gowers does not lay any stress on this complication, although he notes the frequency of meningitis in tumour of the brain, and thinks it may be a factor in the production of papillitis.

The most serious objection which has been urged against this theory, is the fact that well marked papillitis may occur and run its course without any cerebral symptoms, such as would infallibly be the case with so serious a lesion as œedema of the brain.

In the second part, on ophthalmoscopic changes in special diseases, the relations of optic neuritis to tumour of the brain; its symptoms, course, and duration are discussed with more detail.

The general results may be summed up as follows:-That in optic neuritis, consecutive to tumours of the brain, there is no relation between the size, position, and nature of the tumour and the intra-ocular affection. In the present state of our knowledge, it is impossible to say on what the occurrence of optic neuritis depends; but, where meningitis occurs as a complication, neuritis may sometimes be distinctly traced to it. Optic neuritis is essentially transient, and may run its course and pass away, leaving atrophy of the disc, or it may make its appearance only in the later stages of the disease.

"In many cases in which neuritis occurs long after the symptoms of tumour have existed, its occurrence precedes death by no long interval."

The neuritis of tumour is generally double, rarely unilateral. It may exist without affecting the visual functions, and its presence, unless looked for, may not be detected. Its course and duration appear to be influenced by the nature and progress of the morbid growth and its consequences, and probably also in syphilitic cases by the treatment.

"Significance:-The value of optic neuritis as an indication of the existence of an intra-cranial tumour is very great.

* Annales D'oculistique. July and August, 1879. 
Tumour is the cause of the great majority of cases of neuritis due to organic disease, and not the result of toxæmia. On the other hand, neuritis is present, at some period, in at least four-fifths of the cases of tumour, and it may be the only unequivocal sign of organic intra-cranial disease."

After tumour of the brain, acute meningitis is the most frequent cause of intra-ocular inflammation, and although generally less intense than in tumour, may occasionally be so pronounced as to simulate the typical characters of the latter. Dr. Gowers' observations point to basilar meningitis, with extension of the inflammatory process to the trunk of the optic nerve as the exciting cause of the optic neuritis, and that there is no correspondence-as was assumed by Manz and Seber-between the occurrence of neuritis, the existence of increased intra-ocular pressure, and the distension of the sheath. "The latter is probably related solely to an increase of the sub-arachnoid fluid, without which even great distention of the ventricles not only does not cause dropsy of the sheath, but may even remove it."

The researches of Parinand on optic neuritis in the acute meningitis of infancy, so far support the view that hydrocephalus with distention of the sheath of the optic nerve exerts no influence in the production of neuritis; but, on the other hand, he has found distention of the lateral ventricles with or without distention of the sheath of the optic nerve invariably present in optic neuritis.

The number and variety of diseases of the vascular system in which ophthalmoscopic symptoms have been observed is considerable, but on account of the comparative independence of the intra-ocular circulation, many of them have little clinical and almost no diagnostic value. There is, however, one exception-viz., albuminuric retinitis, usually associated with, and generally the first reliable indication of granular kidney. The retinal changes, with the accompanying dimness of vision, generally correspond in time with the cardiac hypertrophy and increased arterial tension which characterise the later stages of the disease.

"The retinal disease presents certain elements which are variously combined in different cases. These are-(1) diffuse slight opacity and swelling of the retina, due to œedema of its substance; (2) white spots and patches of various size and distribution, due for the most part to degenerative processes ; (3) hæmorrhage; (4) inflammation of the intra-ocular end of the optic nerve; (5) the subsidence of inflammatory changes may be attended with signs of atrophy of the retina and nerve." 
From a clinical point of view the degenerative form is the most important, on account of its comparative frequency and the absence of constitutional disturbance in the early stage of the disease. Unfortunately, the disease of the kidneys is too far advanced in the majority of cases when the state of the vision and the ophthalmoscopic examination reveal the true character of the disease. The early recognition of the retinal affection-which probably exists long anterior to the period when the accidental failure of the vision causes the patient to seek advice-might, in some cases, afford valuable aid in the diagnosis and treatment of an otherwise incurable disease, for now and again cases occur in which, from the ophthalmoscopic symptoms and the low specific gravity of the urine, there is reason for suspecting kidney disease, and which apparently get well under treatment.

But this would necessitate the routine use of the ophthalmoscope, the intelligent application of which is not by any means a common accomplishment.

Dr. Gowers very properly insists on the student learning to use the instrument early in his practical work at the hospital.

In a note on ophthalmoscopic micrometry, Dr. Gowers recommends a metal screen in front of the lamp, and a concave mirror in the ophthalmoscope, both for the direct and indirect method, but does not appear to take into account the magnifying power of the mirror and the distortion of the image on the screen from the necessity of using oblique pencils of light. It is a very remarkable fact that, considering the great advances which have been made in physiological optics, we are still in want of reliable measurements of the size of the optic disc and retinal vessels.

The appendix, containing a detailed account of fifty cases, with an atlas of the ophthalmoscopic appearances presented by many of them, is, as the author intended, the basis of the work. As a careful record of facts and observations, frequently confirmed by post-mortem examination, it will always occupy a permanent place in the literature of a subject where facts more than theories are a desideratum. The plates, although for the most part uncoloured, being autotypes of sepia drawings, are peculiarly valuable from the evident care which has been bestowed in the production of details, this method allowing, as the author mentions, of more exact rendering of details. As the plates refer to cases they give great assistance in following the descriptive part.

On the whole, we can cordially recommend this work to the profession as being thoroughly reliable. The author does not 
presume that the appearances presented by the fundus are generally pathognomonic of particular diseases, yet the appearance of such a work will not only popularise the subject, but enable the enquirer to obtain, in a short compass, all that is really known in this important department of medical science.

A Note Book of Solubilities, arranged chiefly for the use of Prescribers and Dispensers. By John Eagle, Member of the Pharmaceutical Society. London: H. K. Lewis. 1880.

THIS little book of 58 pages costs half-a-crown. It deals with subjects of but little interest to many, but for those in search of the kind of information here given it must prove of great service. We have often been struck with the difficulty of procuring accurate and definite information on the solubilities of drugs, even on referring to the best handbooks.

The following extract will show the nature of the book:-

\section{ACID, Salicylic.}

The precipitated acid, 1 in about 600 cold water.

The crystalline acid, 1 in about 750 cold water.

1 in 3 cold rectified spirit.

1 in 2 sulphuric ether.

1 in 5 boiling turpentine.

1 in 150 glycerine at the ordinary temperature.

1 in 50 warm glycerine, remains in solution on cooling.

1 in 5 hot glycerine.

1 in 12 warm olive oil.

Its solubilitity in water is increased by the addition of borax, phosphate of sodium, or citrate of potassium.

On the Bile, Jaundice, and Bilious Diseases. By J. Wickham LeGG, Assistant Physician to St. Bartholomew's Hospital, and Lecturer on Pathological Anatomy in the Medical School. London: H. K. Lewis. 1880.

THIs portly volume, of more than 700 pages, has rather a taking title. When we remember the number of persons who, being otherwise healthy, confess to attacks of "the bile," or "say that they are subject to "bilious" disorders, we feel that this book must meet a widespread want. Particularly is this likely to be felt by those who are conscious of some haziness as to what is wrong with "the bile" in such attacks. The addition of the word "jaundice" to the title adds solidity to the whole; and a glance at the pages of the book, bristling 